\title{
基于粉末本构模型和多体力学仿真的模架集成式 粉末成形设备的设计*
}

\author{
邱 诚 夏伟 \\ (华南理工大学机械与汽车工程学院 广州 510640)
}

\begin{abstract}
摘要: 研制出一种新型的低成本、小体积、高精度的专用模架集成式粉末成形设备。以 MSC.Marc 与 MSC.Adams 软件相结 合提出基于数值模拟的 $1 \mathrm{MN}$ 压机设计方法, 即根据粉末压制试验构建末材料流动应力本构模型; 通过该模型在 MSC.Marc 软件数值计算出所设计压机压制过程的模冲载荷; 在分析模架集成式粉末成形设备各个模板在压制工过程运动特点的基础 上, 建立多体运动学数学方程; 将所得模冲载荷作为边界条件加载到对模架集成式粉末成形设备在压制过程的模拟仿真; 并 在 MSC.Adams 软件中分析各个模板在压制过程中力学状态, 从中分析该设备在实际工况下的可靠性。研制出电液比例阀和 光柾尺相结合的控制系统, 实现对缸同时驱动, 解决不同模板之间的动作协调的问题, 所研制试验样机实现零件轴向压制精 度为 $\pm 0.02 \mathrm{~mm}$ 。实际压制过程所得各模冲载荷与模拟结果相一致, 从而验证粉末成形设备设计所采用的本构模型是准确的, 基于数值模拟进行粉末成形设备设计的方法的正确性。

关键词: 粉末压制 模架集成 成形设备 本构模型 数值模拟

中图分类号: TG37

\section{Design and Manufacture of the Mould-frame Integrated Powder Forming Equipment Based on Constitutive Model of Powder and Simulation of Multi-body Dynamics}

\author{
QIU Cheng XIA Wei
}

(School of Mechanical and Automotive Engineering, South China University of Technology, Guangzhou 510640)

\begin{abstract}
The special powder molding machine integrating mould frame is designed and manufactured with the feature such as low-cost, small size and high-precision. During the course of the design and manufacture for the special powder molding machine, the flow stress constitutive model of powder and the multi-body dynamics model are construct. Furthermore, the 1 MN powder metallurgy press is put forward based on the numerical simulation by combining the software of MSC.Marc and MSC.Adams. The numerical result of punch load is computed by software of MSC.Marc and the obtained result is exerted as a boundary condition for simulating the pressing process of the special powder molding machine. From the numerical result, the mechanical state of each pattern plate is analyzed in software of MSC.Adams so that the reliability of the special powder molding machine is obtained at the actual working condition. The control system combining the electro-hydraulic proportional valve and grating ruler is developed which makes the two contrapositive hydro-cylinders drive at the same time. And the movement coordination of different mould plates is resolved. The axial pressing accuracy of the formed part is $\pm 0.02 \mathrm{~mm}$ manufactured by the test prototype. There is consistentency between the measured punch load and the numerical result during the compaction which demonstrates that the constitutive model used for powder forming equipment design is accurate and the method based on numerical simulation is correct for powder forming equipment design.
\end{abstract}

Key words: Powder compaction Integrated mould-frame Powder forming equipment Constitutive model Numerical simulation

0 前言

粉末成形设备是专为粉末治金结构零件压制

*国家科技重大专项资助项目(2010ZX04004-121)。20130115 收到初稿, 20130705 收到修改稿
成形而设计、制造的设备。粉末成形设备按照加压 方式分为单向、双向及多动作三类，而依据驱动方 式则分为机械式、液压式和机械-液压复合式。其中 机械式的有偏心式、曲轴式、凸轮式、肘节式和转 盘式等。目前国内大部分粉末零件生产商大都采用 该类模架; 液压式模架采用位置闭环控制, 成形精 
度较高, 如华南理工大学研制成功的集成缸驱动模 架, 大幅度提高了零件质量。液压套缸式模架以德 国曼内斯曼公司的产品为代表, 是目前最先进的模 架, 受专利技术保护。

目前国内的粉末治金压机, 往往是采用三梁四 柱式结构, 或者是利用通用型框架式液压机进行改 装。三梁四柱式结构压机的刚性比较差, 精度不能 得到保证。利用通用型框架式液压机改装的粉末冶 金压机, 没有考虑到粉末零件的压制特点, 压制模 式与普通液压机的压制模式相同，不能根据各种不 同粉末的最佳压制曲线来设定压制的动作, 难以达 到最佳的压制效果。

国际上日本良塚精机(株)与日本玉川机械(株) 的粉末成形压机主要是机械式压机, 普遍使用模架, 压制力由 $120 \mathrm{kN} \sim 7.5 \mathrm{MN}$ 不等 ${ }^{[1]}$ 。德国 Mannesmann 开发的用于 HPM.E2 系列压制设备上 的可控模冲模架, 利用 32 位微处理器, 根据位置和 压力可对多达 9 个模冲进行快速、准确的闭环控制, 从而解决了利用机械挡块定位, 因机械挡块变形回 弹而导致压坏产生裂纹等一系列问题 ${ }^{[2-3]}$ 。此模架结 构已经成为压制高精度零件模架的典型代表。德国 KOMAGE 公司开发了具有智能的粉末压制成形设 备, 采用液压闭环控制的液压系统, 利用光栅监测 活塞的位移和传感器监测活塞的液压力。利用计算 机控制活塞的行程、速度和加速度。定程精度可达 $\pm 0.01 \mathrm{~mm}^{[4-5]}$ 。美国 Gasbarre 制品公司生产的粉末 成形压机都是由曲轴驱动, 使用模架的阴模浮动型 机械式压机, 依据成形制品压坏的形状复杂程度分 成不同的系列。其中模架系列压机的特点是模架可 以更换, 但压机具有必要的各种调节功能, 在其给 定范围内都可以进行无级调节, 从而可简化模架与 模具设计。压制能力在 $50 \sim 600 \mathrm{kN}$ 。另外多动作系 列是为压制成形形状复杂的多台面制品压坯而设 计、制造的, 压制能力可达 $7 \mathrm{MN}^{[6]}$ 。德国 Dorst 机 器与设备制造公司的产品有机械式自动粉末成形压 机和液压式自动粉末成形压机等。其中 TPA 系列粉 末成形压机是一种肘杆式压机, 最大吨位达 4.5 $\mathrm{MN}$; TPA-H 系列粉末成形压机是液压式全自动 $\mathrm{CNC}$ 粉末冶金成形压机, 压制能力可达 $8 \mathrm{MN}$;

DACS 系列压机是一类压制位置精度高、高效率的 机械式自动粉末成形压机, 专为满足当前硬质合金、 技术陶瓷及铁氧体工业发展需要而生产 ${ }^{[7]}$ 。

现使用的专用粉末治金压机无论是进口机还 是国产机, 结构过于复杂, 体积庞大, 价格过高, 使得大多数粉末冶金企业望而却步。而且模架都要 根据不同零件进行单独设计, 并存在结构复杂、调
整困难、成形工艺复杂和造价昂贵等缺点。如何在 保证粉末冶金零件压制质量的前提下，降低粉末冶 金压机的成本、减少粉末冶金压机的体积, 成为当 前粉末冶金行业的一大难题。

本文根据典型多台阶复杂零件的粉体成形从 合模开始到压制成形过程的工作情况，提出压机与 模架集成的新型粉末成形设备设计，并基于多体动 力学理论, 应用 ADAMS 软件对该过程进行多体动 力学仿真, 获取了该过程中机架部分动载荷、各类 模板行程及各个约束的约束反力等的变化规律, 通 过虚拟样机的仿真分析和优化结果实现物理样机试 制。同传统压机相比, 它彻底改变了传统设备的结 构, 使得设备结构简单, 调试方便, 能最大限度发 挥粉末成形专用设备的特点, 提高成形精度, 形成 自主知识产权。

\section{1 设计方案提出}

模架集成式粉末成形设备的结构如图 1 所示, 该设备采用四柱式结构, 以保证整体刚度同时实现 受力件和导向件分离, 让导柱专用于导向, 以提高 成形精度。该设备要成形上 2 下 3 的多台面粉末冶 金零件, 因此共有 3 块下模板, 相应设置 3 组驱动 液压缸。上横梁以类似的方法安装了 2 块上模板和 阴模板驱动液压缸。阴模安装在阴模板 10 上, 由于 阴模板可以独立动作, 因此可实现浮动压制。3

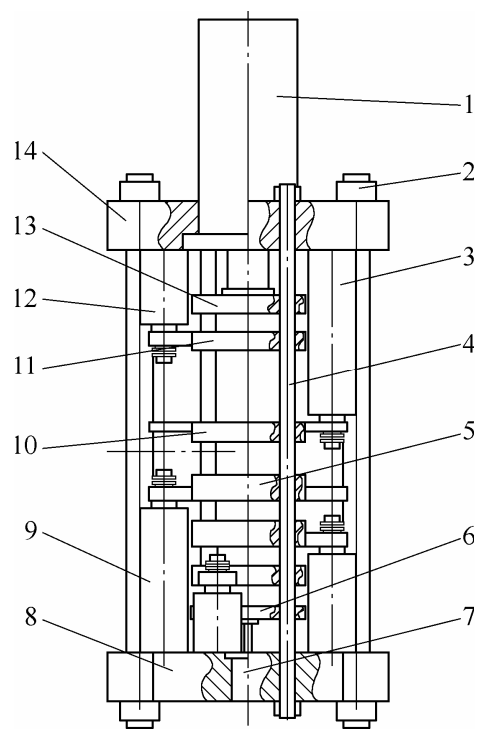

图 1 模架集成式成形设备结构图

1. 上缸 2. 外拉杆 3. 阴模驱动夜压缸 4. 导向拉杆 5. 下一模台 6. 芯棒模台 7. 芯棒驱动液压缸 8 . 下横梁 9. 下一模台驱动液压缸 10. 阴模台 11. 上二模台 12 . 上二模台驱动液压缸 13 . 上一模台 14. 上横梁 
个下模冲分别安装在 3 块下模板上, 各个独立的动 作组合起来可以实现装粉、压制、脱模等动作。2 个上模冲安装在 2 块上模板上, 成形零件的 2 个上 台阶面。芯棒安装在芯棒模板 6 上, 也可以独立运 动, 形成零件的内孔。

\section{2 模板运动分析与基于多体系统动力 学建模}

一般多台面粉末冶金零件的成形工艺过程如 图 2 所示。图 2a 按照零件各台面的装粉量, 模冲和 阴模移动至各自的装粉位置; 图 $2 \mathrm{~b}$ 送料装置送粉, 把粉料送到模腔位置后再进行往复几次来回振动, 使粉末装得更加均匀, 装粉后为了防止上模冲下压 时粉末溅出, 阴模板 10 将向上移动一段距离; 图 $2 \mathrm{c}$ 上模冲通过上模板 11 下移至粉末上平面; 图 $2 \mathrm{~d}$ 依据零件形状, 各模冲之间相互配合移动使粉末在 模腔中重新分布, 此过程中并没有开始进行粉末压 制, 模腔内粉末的体积保持不变; 图 2e 进行压制, 各模冲的具体压制参数由不同零件的压制生产工艺 决定; 图 $2 \mathrm{f}$ 松开上模冲的压力, 让零件产生一定的 回弹，根据实际需要，有时也会保持压力，或者部 分卸压; 图 $2 \mathrm{~g}$ 阴模与所有安装上模冲的模板同时上 行一段距离, 准备零件的脱模; 图 $2 \mathrm{~h}$ 阴模与芯杆下 行; 图 $2 \mathrm{i}$ 分阶段退出中间模冲, 防止脱模时零件回 弹出现裂纹; 图 $2 \mathrm{j}$ 上模冲退回, 零件脱出。

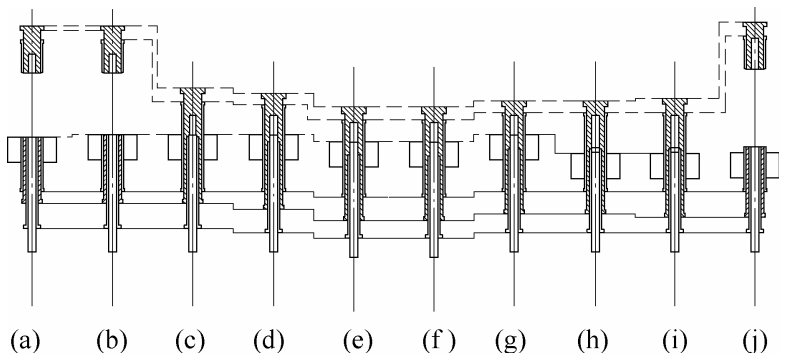

图 2 多台面粉末零件的成形工艺过程

从多台面粉末治金零件的成形工艺过程可以 看出, 压制过程是由设备中不同部件共同运动而构 成。其中导柱可以看作刚体, 阴模和各层模板都看 作柔性体。

首先从多刚体系统入手, 理想条件下多刚体系 统的拉格朗日运动方程 ${ }^{[8-9]}$

$$
\left\{\begin{array}{l}
\frac{\mathrm{d}}{\mathrm{d} t}\left[\frac{\partial K}{\partial \dot{q}_{j}}\right]-\frac{\partial K}{\partial q_{j}}+\sum_{i=1}^{n} \frac{\partial \Phi_{i}}{\partial q_{j}} \lambda_{i}=F_{j} \\
j=1,2, \cdots, 6 \\
\Phi_{i}=0 \quad i=1,2, \cdots, m
\end{array}\right.
$$

式中, $K$ 为动能; $q_{j}$ 为描述系统的广义坐标; $\Phi_{i}$ 为
系统的约束方程; $F_{j}$ 为在广义坐标方向的广义力;

$\lambda_{i}$ 为 $m \times 1$ 的拉格朗日乘子列阵。

对于柔性体, 其上任一点的运动是动坐标系的 刚性运动与弹性变形的合成运动, 即在刚体运动的 基础上，还需要一组坐标来描述柔性体上各点相对 动坐标系的变形, 即共有惯性坐标系和动坐标系, 该坐标系可以相对惯性坐标系进行有限的移动和 转动。

这样柔性体上某点 $p$ 相对惯性系的位置矢量表 示为 ${ }^{[10]}$

$$
\boldsymbol{r}=\boldsymbol{r}_{a}+\boldsymbol{A}\left(\boldsymbol{r}_{p}+\boldsymbol{u}_{p}\right)
$$

式中, $\boldsymbol{r}_{a}$ 为动坐标系下的位置矢量, $\boldsymbol{r}_{p}$ 为动坐标系 原点在惯性坐标系下的位置矢量, $\boldsymbol{u}_{p}$ 为惯性坐标系 原点的位置矢量, $\boldsymbol{u}_{p}$ 为 $p$ 点相对动坐标系的位置矢 量, $\boldsymbol{A}$ 为坐标转换矩阵。对于变形体, $\boldsymbol{u}_{p}$ 可表示为

$$
\boldsymbol{u}_{p}=\boldsymbol{\Phi}_{p} \boldsymbol{q}_{f}
$$

式中, $\boldsymbol{\Phi}_{p}$ 为点 $p$ 满足里兹基矢量要求的假设变形模 态矩阵, $\boldsymbol{q}_{f}$ 为变形的广义坐标。

由式(1)拉格朗日方程导出如下柔性体的运动 方程 ${ }^{[11]}$

$$
\left\{\begin{array}{l}
\frac{\mathrm{d}}{\mathrm{d} t}\left(\frac{\partial L}{\partial \dot{\xi}}\right)-\frac{\partial L}{\partial \boldsymbol{\xi}}+\frac{\partial \Gamma}{\partial \dot{\xi}}+\left(\frac{\partial \psi}{\partial \boldsymbol{\xi}}\right)^{\mathrm{T}} \lambda-Q=0 \\
\psi=0
\end{array}\right.
$$

式中, $\psi$ 为约束方程; $\lambda$ 为对应于约束方程的拉氏 乘子; $\boldsymbol{\xi}$ 为广义坐标, $\boldsymbol{\xi}=\left(\begin{array}{lllllll}x & y & z & \psi & \theta & \phi & q_{i}\end{array}\right)^{\mathrm{T}}=$ $\left(\begin{array}{lll}r & \psi & q\end{array}\right)^{\mathrm{T}}(i=1,2, \cdots, m) ， Q$ 为投影到 $\boldsymbol{\xi}$ 上的广义 力; $L$ 为拉格朗日项, 定义为 $L=T-W, T$ 和 $W$ 分 别表示动能和势能; $\Gamma$ 表示能量损耗函数, $\dot{\xi}$ 为在 系统广义坐标 $\xi$ 中的时间导数。并由式(5)、(6)、(8) 表示 ${ }^{[12]}$

$$
T=\frac{1}{2} \dot{\xi}^{\mathrm{T}} \boldsymbol{m}(\boldsymbol{\xi}) \dot{\xi}
$$

式中, $\boldsymbol{m}(\boldsymbol{\xi})$ 为质量矩阵。

$$
W=W_{g}(\boldsymbol{\xi})+\frac{1}{2} \xi^{\mathrm{T}} \boldsymbol{k} \boldsymbol{\xi}
$$

式中， $\boldsymbol{k}$ 是对应于模态坐标 $q$ 的结构部件的广义刚 度矩阵, 通常为常量。由式(2)、(3)可知, 重力势能 $W_{g}$ 表示为 ${ }^{[12]}$

$$
\begin{gathered}
W_{g}=\int_{W} \rho \boldsymbol{r}_{q} \cdot g \mathrm{~d} W=\int_{W} \rho\left[\boldsymbol{r}_{a}+A\left(\boldsymbol{r}_{P}+\boldsymbol{\Phi}_{P} \boldsymbol{q}_{f}\right)\right]^{\mathrm{T}} g \mathrm{~d} W \\
\Gamma=\frac{1}{2} \dot{\boldsymbol{q}}^{\mathrm{T}} \boldsymbol{D} \dot{\boldsymbol{q}}
\end{gathered}
$$


式中, $\boldsymbol{r}_{q}$ 为广义坐标矢量, $\rho$ 为柔性体密度, $g$ 为 重力加速度, $\dot{\boldsymbol{q}}$ 为在系统广义坐标 $\boldsymbol{q}$ 中的时间导数, 矩阵 $\boldsymbol{D}$ 为包含阻尼系数 $c$ 的常值对称阵。

将求得的 $T, W, \Gamma$ 代入式(4), 得到最终的运动 微分方程为 ${ }^{[12]}$

$$
\begin{gathered}
\boldsymbol{m} \ddot{\boldsymbol{\xi}}+\dot{\boldsymbol{m}} \dot{\boldsymbol{\xi}}-\frac{1}{2}\left(\frac{\partial \boldsymbol{m}}{\partial \boldsymbol{\xi}} \dot{\boldsymbol{\xi}}\right)^{\mathrm{T}} \dot{\boldsymbol{\xi}}+\boldsymbol{k} \boldsymbol{\xi}+f_{g}+ \\
\boldsymbol{D} \dot{\boldsymbol{\xi}}+\left(\frac{\partial \psi}{\partial \boldsymbol{\xi}}\right)^{\mathrm{T}} \lambda=Q
\end{gathered}
$$

式中, $\boldsymbol{m}, \boldsymbol{m}$ 为柔性体的质量矩阵及其对时间的导 数; $f_{g}$ 为柔性体的重力, $\partial \boldsymbol{m} / \partial \boldsymbol{\xi}$ 为质量矩阵对柔性 体广义坐标的偏导数, 它是一个 $(M+6) \times(M+6) \times$ $(M+6)$ 维张量, $M$ 为模态数。

\section{3 压制过程粉末材料本构建模}

在压制过程中由于相对密度变化发生变化, 导 致材料产生几何硬化。由于基体材料力学性能发生 变化, 导致材料产生应变硬化。这两类硬化都将影 响粉末材料的流动应力模型。

粉末材料压制时的流动应力模型可由式(10) 表示

$$
\sigma_{R}^{2}=\delta(\rho) \sigma_{0}^{2}
$$

式中, $\sigma_{R}$ 代表当粉末材料相对密度为 $\rho$ 时的屈服应 力, $\delta$ 代表粉末材料单向拉伸屈服应力与致密金属 单向拉伸屈服应力之间的系数, 而 $\sigma_{0}$ 则代表了无孔 隙(致密)材料的屈服应力。

$\mathrm{KUHN}$ 等 ${ }^{[13]}$ 假设粉末材料的流动应力与致密 材料的相同, 即

$$
\delta=1
$$

在 LEE 等 ${ }^{[14]}$ 的屈服条件中, 提出

$$
\delta(\rho)=\frac{\left(\rho-\rho_{c}\right)^{2}}{\left(1-\rho_{c}\right)^{2}}
$$

式中, $\rho_{c}$ 是屈服应力为 0 时的相对密度, 与松装相 对密度相近, 但不一定相等。DORAIVELU 等 ${ }^{[15]}$ 则提出

$$
\delta(\rho)=\frac{\rho^{2}-\rho_{c}^{2}}{1-\rho_{c}^{2}}
$$

试验在 SANS-CMT5105 试验机上进行, 采用 常用雾化铁粉, 混合少量有机润滑剂进行压制, 该 混合粉末的初始相对密度为 0.42 。压制模具直径为 $12 \mathrm{~mm}$, 装粉高度为 $20 \mathrm{~mm}$, 上模冲的最大压制力
设定为 $75 \mathrm{kN}$ 。在压制过程中, 试验机自动记录上 模冲的压力和行程曲线即压制曲线。将采用不同流 动应力模型模拟获得的压制曲线和试验获得的压制 曲线进行比较(图 3)。

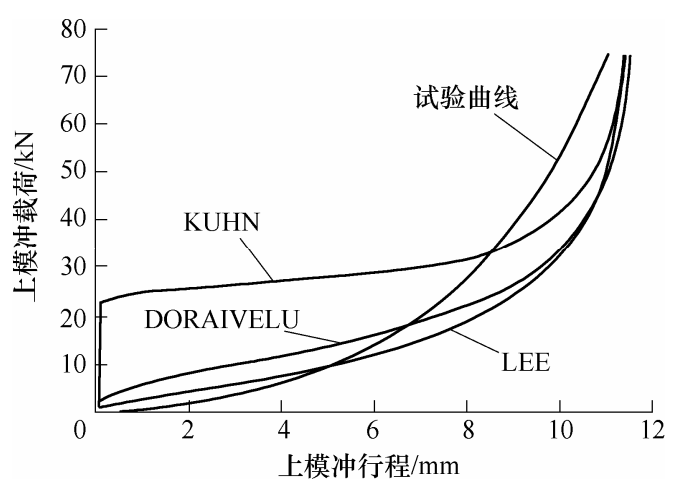

图 3 试验压制曲线和模拟压制曲线的对比

从图 3 中可以看出试验和以 KUHN、 DORAIVELU 和 LEE 的流动应力模型模拟得到的 上模冲载荷-行程曲线的变化趋势相近。图 3 中曲线 表明, 粉末致密化主要发生在载荷施加的初期阶段, 此阶段粉末密度较低, 颗粒之间结合区较小, 易于 发生塑性变形, 同时相互间的间隙也比较大, 结合 力很弱, 这些因素导致粉末很容易产生屈服和流动, 此阶段粉末的流动应力远远低于致密金属的流动 应力。

将试验获得的压力-行程曲线进行整理, 与模拟 结果进行对比校正, 考虑到 DORAIVELU 对 $\delta(\rho)$ 的 量纲分析, 黄春曼等 ${ }^{[16-17]}$ 提出了以下的流动应力 模型

$$
\delta(\rho)=\frac{\rho^{9}-\rho_{c}^{9}}{1-\rho_{c}^{9}}
$$

即

$$
\sigma_{s P}=\frac{\rho^{9}-\rho_{c}^{9}}{1-\rho_{c}^{9}} \sigma_{S H}
$$

式中, $\sigma_{s P}$ 为粉末材料相对密度为 $\rho$ 时的流动应力, $\sigma_{S H}$ 为初始松装粉末硬化后的流动应力, 试验测定 为 $400 \mathrm{MPa}$ 。

\section{4 数值模拟与结果分析}

应用前面推导的本构关系，作者利用商业有限 元软件 MSC.Marc 中所提供的用户编程接口, 开发 了粉末力学模型的通用用户接口计算模块, 用该程 序模拟多台阶面的上 2 下 3 结构的典型零件压制过 程, 典型零件尺寸如图 4 所示, 粉末体的初始松装 尺寸为 $H_{1}=50 \mathrm{~mm}, H_{2}=12.5 \mathrm{~mm}, H_{3}=25 \mathrm{~mm}$, 
建立了 $1 / 4$ 该圆柱体的三维模型。模型包括 1260 个六面体单元, 分析采用全积分六面体单元(在 MSC.Marc 中为 7 号单元), 模具均假设为刚性体。 压制模型尺寸及网格划分如图 5 所示。3 个上模冲 以 $5 \mathrm{~mm} / \mathrm{s} 、 1.25 \mathrm{~mm} / \mathrm{s} 、 2.5 \mathrm{~mm} / \mathrm{s}$ 的速度压下, 成形 时间为 $6 \mathrm{~s}$, 得出各个模冲载荷曲线的模拟结果(图 6)。

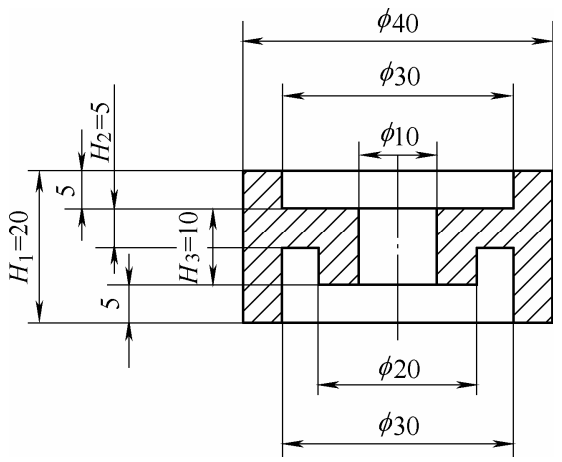

图 4 典型零件图

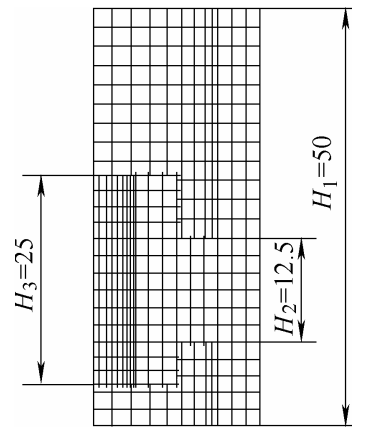

图 5 试样模型尺寸及网格划分

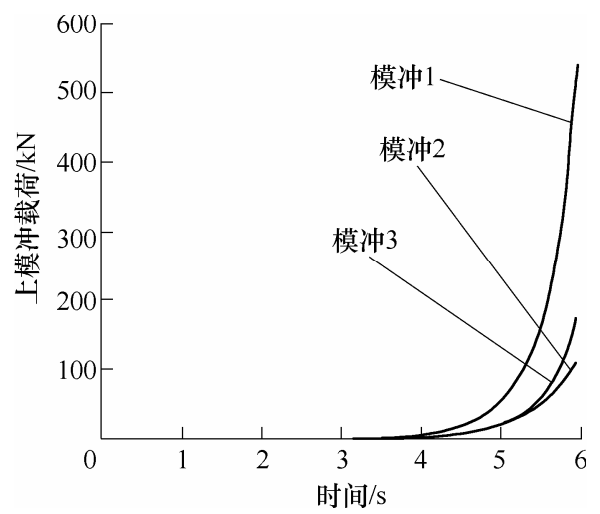

图 6 典型零件各个模冲载荷随时间变化曲线

\section{5 虚拟样机分析}

本文在 Pro/Engineer 软件平台上构建粉末成形 设备三维模型, 然后导入 Hyperworks 软件对设备构 件进行柔性化处理, 生成柔性体并重新置于 ADAMS 中, 来建立粉末成形设备的多柔体系统(图 7), 其中柔性体的总的节点数为 89678 , 总的单元 数为 379865 。将图 6 所得模冲压力曲线加载到虚拟
样机, 对样机进行压制过程的力学分析。图 8 为压 制过程中各个模板应力分布云图，其中模板 1 和 6 应力最大，其最大应力分别达到 $211 \mathrm{MPa}$ 和 215 $\mathrm{MPa}$ ，如图 9、10 所示。

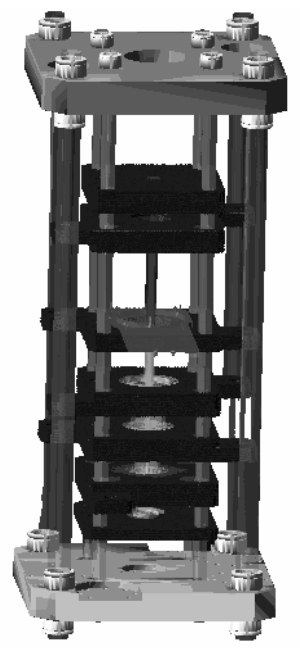

图 7 整机柔性体有限元模型

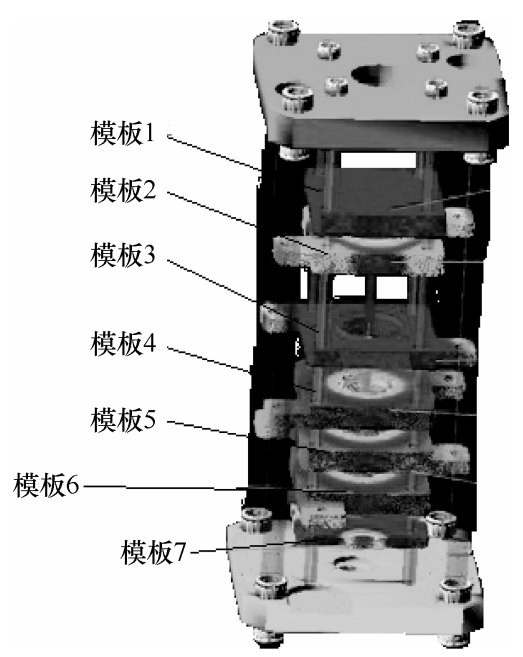

图 8 模板应力分布云图

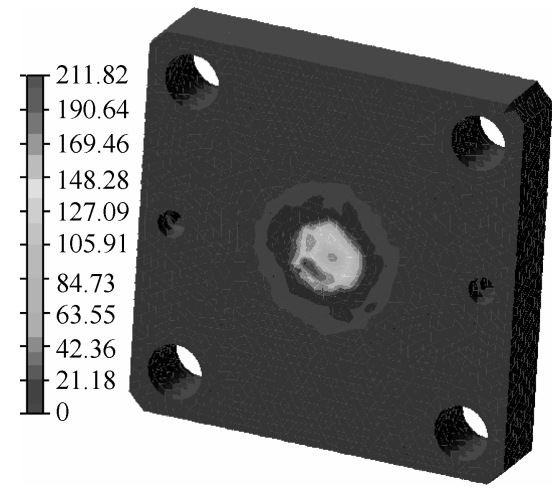

图 9 模板 1 应力分布云图(MPa)

将模板 1、6 的应力结果导入 ANSYS 软件进行 按 2 次 $/ \mathrm{min}$ 的成形频率进行疲劳分析, 其模板 1 和 6 各个部位的疲劳应力循环次数分布如图 11、12 所 示, 由图 11、12 可知模板各部位应力循环次数均大 


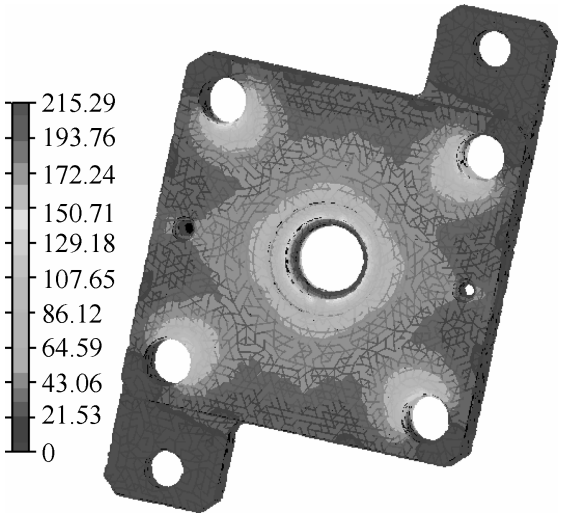

图 10 模板 6 应力分布云图(MPa)

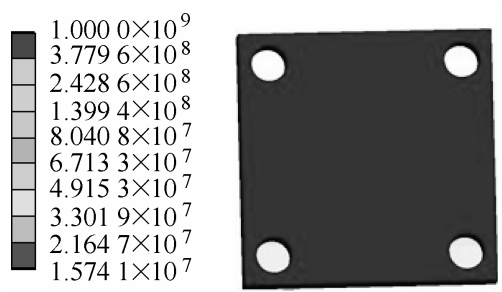

图 11 模板 1 疲劳应力循环次数分布云图

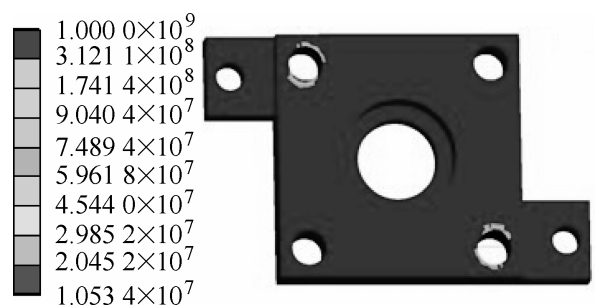

图 12 模板 6 疲劳应力循环次数分布云图

于 $10^{7}$, 符合设计要求。把各模板的整体高度及变 形量及其比值列于表 1 。从表 1 可以看出, 模架结 构的刚度符合设计要求。

表 1 模板刚度校核

\begin{tabular}{ccccc}
\hline 模板 & $\begin{array}{c}\text { 最大变形 } \\
\text { 量 } / \mathrm{mm}\end{array}$ & $\begin{array}{c}\text { 整体高度/ } \\
\mathrm{mm}\end{array}$ & $\begin{array}{c}\text { 最大变形量 } \\
\text { 与整体高度比值 }\end{array}$ & $\begin{array}{c}\text { 比值是否小于 } \\
1 / 500\end{array}$ \\
\hline 模板 1 & 0.030 & 80 & $3.75 \times 10^{-4}$ & 是 \\
模板 2 & 0.010 & 80 & $1.25 \times 10^{-4}$ & 是 \\
模板 3 & 0.004 & 60 & $6.67 \times 10^{-5}$ & 是 \\
模板 4 & 0.007 & 80 & $8.75 \times 10^{-5}$ & 是 \\
模板 5 & 0.006 & 80 & $7.50 \times 10^{-5}$ & 是 \\
模板 6 & 0.040 & 80 & $5.00 \times 10^{-4}$ & 是 \\
模板 7 & 0.008 & 80 & $1.00 \times 10^{-4}$ & 是 \\
\hline
\end{tabular}

\section{6 液压系统设计}

根据压机本体结构以及使用浮动压制成形，使 用两台压力补偿变量洜分两套相对独立的回路。分 别控制相应的液压缸。考虑到同时动作的可能性, 一个回路控制上内驱动缸、下中驱动缸、下外驱动 缸、送料缸, 芯棒驱动缸。另一个回路驱动上驱动
缸、阴模驱动缸、上外驱动缸。粉末冶金产品高度 方向的尺寸精度是由压机定位精度决定的, 本压机 采用吸入式装粉, 粉末治金产品零件的最终重量便 取决于阴模以及各模冲的定位精度, 尤其对盘状零 件的影响较大。因此对两个回路均用电液比例阀进 行调速, 配合光栅位移测量和控制系统实现位移闭 环控制。为方便调节个浮动环节的浮动力的大小, 在阴模、下外模台、下中模台、下内模台相应的回 路均设有比例压力阀, 配合压力传感器实现压力调 节的闭环控制。在双缸驱动上用分流阀实现双缸同 步, 解决了同步驱动的问题, 避免由于不同步很容 易导致导向部分零件受额外的应力, 减小导向零件 的磨损。系统原理图如图 13 所示。

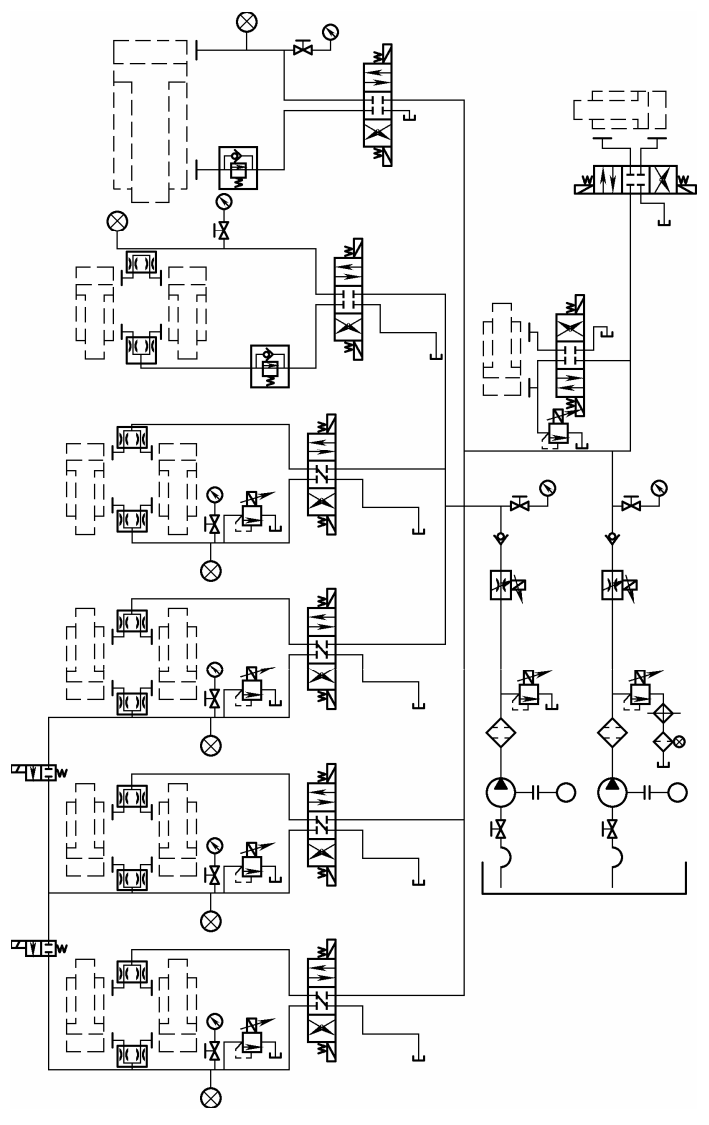

图 13 液压系统原理图

\section{7 控制系统设计}

控制系统采用上下位机控制, 上位机为普通计 算机, 下位机用三菱 FX-2N 可编程序控制器, 通过 串口通信交换数据。

控制系统结构如图 14 所示, 产品的数据输入及 修改通过键盘输入。位移测量使用光㮽尺, 总共需要 7 条光栅尺, 用 4 个通道 ISA 卡两块装在微机上实现 位移测量, 实现零件轴向压制精度可达 $\pm 0.02 \mathrm{~mm}$ 。 


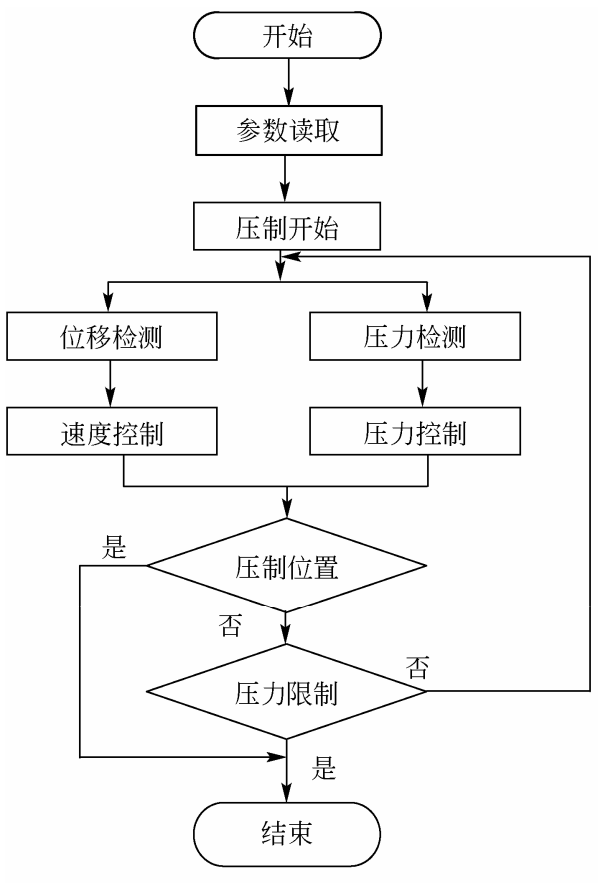

图 14 控制流程图

本设备需要对每个模冲的成形压力进行测量。 共设有 7 个压力传感器。通过模拟量输入模块输入 到可编程序控制器。开关信号直接输入可编程序控 制器。

用于控制的比例阀有 9 个, 可编程序控制器通 过模拟量输出模块驱动相应的比例放大电路推动相 应的比例阀。开关信号由可编程序控制器直接输出 给相应的部件。

研制成功的模架集成式压制设备见图 15, 该设 备的主要参数见表 2 。该设备的结构已申请发明专 利。结合对图 4 多台阶面的上 2 下 3 结构的典型零 件压制试验, 得出模拟结果与试验结果对比如图 16 所示。可以看出采用本文提出的流动应力模型的模 拟结果与试验结果较为一致。该模型能较准确地指 导新型压机进行结构设计。

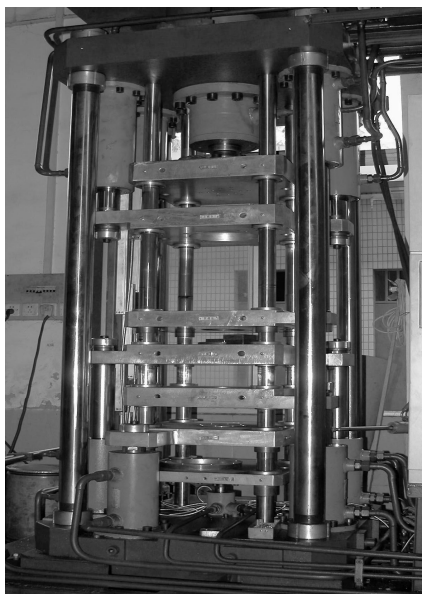

图 15 模架集成式压制设备
表 2 模架集成式压制设备机械参数

\begin{tabular}{lcc}
\hline 名称 & 压力 $/ \mathrm{kN}$ & 行程 $/ \mathrm{mm}$ \\
\hline 上内模板 & 630 & 400 \\
上外模板 & 500 & 400 \\
阴模板 & 400 & 150 \\
下外模板 & 400 & 80 \\
下中模板 & 400 & 80 \\
下内模板 & 400 & 80 \\
芯棒模板 & 50 & 150 \\
送料缸 & 20 & 300 \\
\hline
\end{tabular}

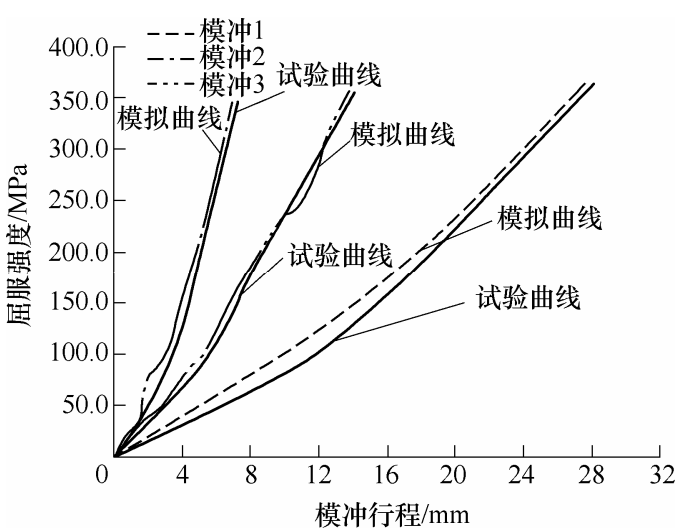

图 16 试验屈服强度-行程曲线和本文提出的模型 模拟屈服强度-行程曲线的对比

\section{8 结论}

将粉末成形的本构模型和刚柔耦合多体动力 学模型应用在集成式粉末成形设备的系统设计中, 将各个模板作为柔性体，建立了集成式粉末成形设 备系统刚柔耦合动力学模型, 对粉末成形过程中系 统的动态特性进行了仿真, 同时对液压和控制系统 等关键技术进行改进, 开发出新型试验样机并得出 如下结论。

(1) 研制出一种全新的、具有自主知识产权的 新型粉末成形设备, 它彻底改变传统设备的结构, 实现压机与模架集成化设计, 使得设备结构简单, 调试方便，样机也制造完毕，并投入使用。

（2）通过将粉末材料流动应力本构模型与多体 动力学模型相结合, 实现压制过程的模拟仿真, 对 高精度粉末压制设备各部件的设计都进行了深入的 研究, 并形成了新型粉末成形设备的设计方法。改 变传统粉末压制设备的设计模式。

(3) 研制出电液比例阀和光栅尺相结合的控制 系统, 实现了对缸同时驱动, 解决不同模板之间 的动作协调的问题, 实现了零件轴向压制精度为 $\pm 0.02 \mathrm{~mm}$ 。 


\section{参 考 文 献}

[1] GETHIN D T, TRAN D V, LEWIS R W, et al. An investigation of powder compaction processes[J]. Journal of Powder Metallury, 1994(30): 380-385.

[2] RAINER L, ANDREAS Z. Adapter system boosts mannesmamm's presses[J]. Metal Powder Report, 1994, 49(10): 24-27.

[3] 董建东, 熊晓红, 黄树槐. 粉末冶金压机的应用与进展 [J]. 粉末治金工业, 1998, 8(2)：29-31.

DONG Jiandong, XIONG Xiaohong, HUANG Shuhuai. Applications and progress of PM presses[J]. Powder Metallurgy Industry, 1998, 8(2): 29-31.

[4] NORBERT N, MANNESMANN D A G. Die filling and powder transfer in powder compacting[C/CD]//1996 PM World Congress, Washington, 1996.

[5] 罗鸣. 先进的压制技术与具有智能的德国柯美佳压机 (KOMAGE)[J]. 粉末治金工业, 1995(6)：259-263.

LUO Ming. Advanced pressing technology and an intelligent Germany KOMAGE pressing machine[J]. Powder Metallurgy Industry, 1995(6): 259-263.

[6] 罗宗强, 刘华, 周玉山. 粉末冶金压力机的发展现状与 展望[J]. 机械工艺师, 2000, 241(10): 56-57.

LUO Zongqiang, LIU Hua, ZHOU Yushan. Powder metallurgy press development present situation and prospect[J]. Machinery Manufactruing Engineer, 2000, 241(10): 56-57.

[7] DORST. Tpa 6/2 universalpress[EB/OL]. [2012-04-12]. http: //www.dorst.de/dorst_seite/index.html.

[8] ZHU Chunxia, ZHU Lida, LIU Yongxia, et al. Dynamics modeling and co-simulation of rigid-flexible coupling system of 3-TPT parallel $\operatorname{robot}[\mathrm{C}] / /$ Proceedings of the IEEE International Conference on Automation and Logistics 2007, Jinan, China, 2007.

[9] CARRERA E, SERNA M A. Inverse dynamics of flexible robots $[\mathrm{J}]$. Mathematics and Computers in Simulation,
1996(41): 485-508.

[10] ZHONG X, YANG R Q, XU Z F, et al. Dynamic modeling of multi-Flexible system-theory and application[J]. Mechanical Science and Technology, 2002(21): 387-389.

[11] SIMEON B. On lagrange multipliers in flexible multibody dynamics[J]. Computer Methods in Applied Mechanics and Engineering, 2006(195): 6993-7005.

[12] Mechanical Dynamics Inc. ADAMS/FLEX user's manual[S]. Los Angeles: Mechanical Dynamics Inc, 2004.

[13] KUHN H A, DOWNEY C L. Deformation characteristics and plasticity theory of sintered powder materials[J]. International Journal of Powder Metallurgy, 1971, 7(1): 15-25.

[14] LEE D N, KIM H S. Plastic yield behaviour of porous metals[J]. Powder Metallurgy, 1992, 35(4): 275-279.

[15] DORAIVELU S M, GEGEL H L, GUNASEKERA J S, et al. A new yield function for compressible $\mathrm{P} / \mathrm{M}$ materials [J]. International Journal of Mechanical Sciences, 1984, 26: 527-535.

[16] 黄春曼. 粉末精密成形装备与工艺的研究[D]. 广州: 华 南理工大学, 2006.

HUANG Chunman. The equipment and process research of powder precision compaction[D]. Guangzhou: South China University of Technology, 2006.

[17] 陈普庆. 金属粉末压制过程的力学建模和数值模拟[D]. 广州: 华南理工大学, 2004.

CHEN Puqing. Mechanical modeling and numerical simulation of the metal powder compaction process[D]. Guangzhou: South China University of Technology, 2004.

作者简介: 邱诚, 男, 1973 年出生, 博士研究生。主要研究方向为粉末 冶金精密成形设备及工艺。

E-mail: cqiu@scut.edu.cn 